Cahiers de philosophie de l'université de

\title{
La perception des rapports. Un nœud qui se défait
}

\author{
Marian Hobson
}

\section{(2) OpenEdition}

Journals

Édition électronique

URL : https://journals.openedition.org/cpuc/608

DOI : $10.4000 /$ cpuc.608

ISSN : 2677-6529

Éditeur

Presses universitaires de Caen

\section{Édition imprimée}

Date de publication : 31 décembre 2014

Pagination : 11-22

ISBN : 978-2-84133-512-1

ISSN : 1282-6545

Référence électronique

Marian Hobson, «La perception des rapports. Un nœud qui se défait », Cahiers de philosophie de l'université de Caen [En ligne], 51 | 2014, mis en ligne le 13 juin 2018, consulté le 01 février 2023. URL http://journals.openedition.org/cpuc/608; DOI : https://doi.org/10.4000/cpuc.608

Creative Commons - Attribution - Pas d'Utilisation Commerciale 4.0 International - CC BY-NC 4.0 https://creativecommons.org/licenses/by-nc/4.0/ 


\title{
La perception des rapports. Un nœud qui se défait
}

\begin{abstract}
T e Diderot des annéEs 1740 - il a 30 ans en 1743 - est tout ensemble tâtonnant et plein d'énergie. Je ferai d'abord un bref historique de l'esthétique de ce Diderot-là, celui qui commence sa carrière d'intellectuel, de publiciste, d'écrivain et de philosophe. Ensuite, j'élargirai la portée de ces remarques, afin de réfléchir sur un problème méthodologique capital qu'on rencontre dans l'étude de Diderot, dans son esthétique certainement et sans doute dans d'autres domaines. Le problème est celui-ci: a-t-on raison de chercher une structure intellectuelle autre que contextuelle et réactionnelle dans ses idées foisonnantes? A-t-on raison d'y chercher une raison autre que locale? Ou peut-être - je voudrais au moins tester un moment la proposition suivante - ne retrouverait-on pas dans les années 1760 les préoccupations esthétiques du Diderot néophyte, mais à un niveau plus profond, moins thématique?
\end{abstract}

Comme je le laisse entendre dans mon titre, ma réflexion se centre sur l'expression "perception des rapports». Elle se trouve chez Diderot dès la fin des années 1740 et au début des années 1750, jusqu'à l'article "Beau» dans le volume II de l'Encyclopédie, en janvier 1752. Elle a quelque peu l'apparence d'un slogan, dans son esthétique comme dans celle de l'époque, car c'est une phrase venue d'ailleurs et mise en œuvre, plutôt que générée par une réflexion autonome et personnelle. Il faut ajouter des caveat à cette remarque: le slogan est sans doute proposé comme un des points de ralliement de tout un groupe d'intellectuels lié à l'Encyclopédie - non seulement Diderot, mais Briseux, d'Alembert et Dumarsais. Briseux est un théoricien de l'architecture sans renom mais connu de d'Alembert, puisque ce dernier s'y réfère plus d'une fois dans le grand Dictionnaire. Il se place, de son propre aveu, dans la mouvance du grand compositeur Rameau, avec 
qui d'Alembert travaille au début des années $1750^{1}$. D'Alembert a aussi eu des contacts suivis avec Dumarsais ${ }^{2}$, logicien et linguiste, qui fournit les articles sur la langue pour l'Encyclopédie jusqu'à sa mort en 1756, et qui se sert également de l'expression ${ }^{3}$. Ce mot de passe, si c'en est un, ne nous fournit pas une clé pour résoudre les difficultés que l'on peut trouver dans l'esthétique du Diderot de l'époque des années 1740-1752, ne serait-ce que parce qu'il pose un problème majeur supplémentaire: l'expression n'a pas de survie évidente. Elle semble presque disparaître du discours de Diderot - et du public - déjà au milieu des années 1750. Laissée tout simplement pour compte, mais l'a-t-on vraiment abandonnée? Diderot change-t-il vraiment d'optique? En posant ce problème de méthode, on doit se demander si le slogan n'est pas devenu souterrain, tel un chemin qui se perd dans la forêt; on pourrait se demander s'il n'est pas devenu comme une trajectoire de la pensée plutôt qu'un thème.

L'expression "perception des rapports " prend son origine à la fois dans les mathématiques et dans la linguistique contemporaines. Mais je serai obligée ici de laisser de côté la question de l'influence de la linguistique de Dumarsais sur la réflexion esthétique de Diderot. Diderot publie en 1748 des Mémoires sur différents sujets de mathématiques; le premier des cinq textes constituant ces Mémoires porte sur l'acoustique (il sera désormais nommé «Mémoire»). Il le base, dit-il, sur des travaux d'Euler, publiés dans le Tentamen novoe musica de 1739. Diderot fait de la perception des rapports du son, l'origine du plaisir musical. Mais aussitôt, il généralise: «Le plaisir, en général, consiste dans la perception des rapports: ce principe a lieu en poésie, en peinture, en architecture, en morale, dans tous les arts et dans toutes les sciences ${ }^{4}$. D'emblée une objection fait surface. Diderot demande: "La connaissance de ces rapports accompagne-t-elle donc toujours la sensation? "5. Il esquisse une réponse possible: cette

1. Charles-Étienne Briseux (1680-1754) est l'auteur d'un Traité du beau essentiel dans les arts, appliqué particulièrement à l'architecture (1752) et d'un Traité des proportions harmoniques (sans date). C. Girdlestone, Jean-Philippe Rameau: sa vie, son cuvre [1957], Paris, Desclée de Brouwer, 1983.

2. Charles Chesneau Dumarsais, 1676-1756. Son éloge, par d'Alembert, se trouve en tête du volume VII de l'Encyclopédie.

3. Je renvoie le lecteur intéressé à mon article «Ni "mot propre" ni "objet tu": Diderot et la "perception des rapports" ", in Le Mot propre et la périphrase: du tour précieux à l'«objet tu» (Actes du colloque organisé par le Centre interdisciplinaire d'étude des littératures d'Aix-

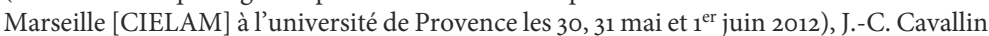
et J.-D. Mazaré (dir.), Paris, Classiques Garnier, 2014.

4. D. Diderot, Mémoires sur différents sujets de mathématiques, in Euvres complètes, R. Lewinter (éd.), Paris, Le Club français du livre, t. II, 1969, p. 39. Cette édition des Euvres complètes de Diderot sera désormais désignée par la mention LEW suivie de la tomaison.

5. Ibid., p. 41. 
connaissance ne serait-elle pas inconsciente, comme le jugement de la distance que fait l'œil, qui opère par « une espèce de trigonométrie naturelle et secrète ${ }^{6}$ ? Si oui, si elle passe effectivement en dessous du radar de la conscience, une analogie entre les lois de la vision et celles de l'ouïe doit exister. Mais immédiatement, Diderot manifeste ce qui pourrait être une réticence: «nous ne déciderons rien là-dessus » 7 .

Dans les dernières pages du «Mémoire», il est fait mention de Rameau, de son ouvrage intitulé Génération harmonique, ou Traité de musique théorique et pratique daté de 1737 et de son "admirable système de composition ${ }^{8}$. Diderot en explique la base: un son ne frappe jamais seul nos oreilles; on entend avec lui d'autres sons concomitants, qu'on appelle ses harmoniques ${ }^{9}$. Diderot parle des "obscurités qui [...] enveloppent le système $»^{10}$ de Rameau, pour suggérer qu'une coopération avec lui serait utile, moins pour augmenter la gloire de l'inventeur que pour aider tout le monde à comprendre le système et pour faire avancer «les progrès de la science des sons " ${ }^{11}$. C'est là une manière quelque peu grinçante d'appeler à un travail en commun. On peut conjecturer que Diderot, sans doute, comme d'Alembert certainement, essaye de coopter le compositeur dans la mouvance de l'Encyclopédie. Si c'est le cas, c'est une tentative qui n'aboutit pas et qui était peut-être vouée dès le début à l'échec: Rameau, célèbre pour ses ressentiments hargneux comme pour son avarice, était en voie de transformer, à l'instar de Pythagore, sa théorie de la musique en une explication du cosmos. D'Alembert, en mathématicien, physicien et sceptique, ne pouvait guère prendre au sérieux ce côté mégalomane et universalisant du système.

Partant des travaux du physicien Sauveur (1653-1716), Rameau a proposé une base physique sur laquelle seraient fondées toutes les pratiques contemporaines de la composition. Une corde, lorsqu'elle vibre, rend un son; ce son est composé du son lui-même, de son octave supérieure, de la douzième et de la dix-septième majeure, et des autres sons harmoniques. Rameau donne ainsi une allure plus scientifique aux lois de la composition et de l'harmonie, jusqu'alors tenues par la plupart des musiciens pour des règles arbitraires, développées uniquement par la tradition et la pratique.

Rousseau, quant à lui, avait subi des vexations dans sa propre tentative de coopération avec le grand compositeur. Il avait arrangé la musique de
6. Ibid.
7. Ibid.
8. Ibid.
9. Ibid., p. 51.
10. Ibid., p. 41
11. Ibid. 
La Princesse de Navarre en 1745, lorsque cet opéra de cour - musique de Rameau, livret de Voltaire - avait été transféré à l'Opéra. Rameau était notoirement mauvais coucheur. Il avait déjà fait dire à Voltaire, auteur du livret, dans un accès d'irritation amusée, que «[Rameau] me mande que j'aye à mettre en quatre vers tout ce qui est en huit et en huit tout ce qui est en quatre ${ }^{12}$. Rousseau, de son côté, a surtout été blessé par le discours désobligeant tenu par Rameau sur la musique d'un intermède, Les Muses galantes, dont il s'était fendu la même année ${ }^{13}$. Chargé par Diderot depuis à peu près la même date, de rédiger la plupart des articles de l'Encyclopédie sur la musique, il ne manque désormais que peu d'occasions pour remettre en question le bien-fondé des théories de Rameau. Celui-ci ne voyait que la haine dans ces articles; en réalité, l'attitude de Rousseau, si elle était guidée par ses mauvaises relations passées avec le compositeur, avait un fondement plus noble que le ressentiment et beaucoup plus intéressant.

Pour Rameau, consonance et dissonance dans la musique renvoient à une «basse fondamentale», dispositif qui n'est peut-être pas actualisé dans la musique, mais qui représente la structure inaudible qui sous-tend une composition. Cette structure est «exprimée " pour ainsi dire (c'est là une métaphore génétique que j'emploie) dans la musique qu'on entend effectivement. Rousseau, en revanche, nie que l'ordre audible dans la musique puisse avoir comme contrepartie cette structure intellectuelle. Selon lui, on ne peut absolument pas postuler, comme le fait Rameau, quelque ordre inaudible aux oreilles quoique perçu par l'intellect, ordre qui soutiendrait l'harmonie. Pour Rousseau, l'harmonie accompagne le son. Sa structure n'est pas inaudible, abstraite et intellectuelle, mais présente et incorporée dans la perception. Rousseau subvertit le raisonnement de Rameau de la façon suivante:

Le musicien ne considère pas le corps sonore en lui-même, il ne le considère qu'en action. Or qu'est-ce que le corps sonore en action? C'est le son. L'harmonie représente donc le son. Mais l'harmonie accompagne le son; le son n'a donc pas besoin qu'on le représente puisqu'il est là ${ }^{14}$.

12. Voltaire, Correspondance générale de M. de Voltaire, Paris, J.-F. Bastien, 1796, t. II, lettre à Hénault du 14 septembre 1744, p. 32.

13. «L'ouvrage a été exécuté plusieurs fois avec assez de succès, savoir en 1745 devant M. le Duc de Richelieu qui le destinoit pour la Cour, en 1747 sur le théâtre de l'Opéra [...]. Ce fut même sur l'exécution de quelques morceaux que j'en avait fait répéter chez $\mathrm{M}$. de la Popelinière que M. Rameau qui les entendit conçut contre moi cette violente haine dont il n'a cessé de donner des marques jusqu'à sa mort »; J.-J. Rousseau, "Avertissement » aux Muses galantes, in Euvres complètes, B. Gagnebin et M. Raymond (éd.), Paris, Gallimard (Bibliothèque de la Pléiade), t. II, 1961, p. 1051. Cette édition des CEuvres complètes de Rousseau sera désormais désignée par la mention $O C$ suivie de la tomaison.

14. J.-J. Rousseau, Examen de deux principes avancés par M. Rameau, in OC V, 1995, p. 360 (je souligne). 
Ce refus de la représentation fait d'ailleurs partie intégrante de sa pensée politique. On remarque ici l'étonnante cohérence de la pensée de Rousseau. En fait, la subordination de l'harmonie à la mélodie, conception développée par Rousseau dans sa Lettre sur la musique française (1753) et dans sa lutte contre Rameau, a eu des effets considérables sur la pratique de la composition musicale, surtout dans l'opéra-comique ${ }^{15}$. Ce changement auquel Rousseau, dans sa théorie, prête un sérieux philosophique, ménage à la musique française comme une sortie de l'esthétique baroque.

La tentative, menée par d'Alembert, de rendre la théorie de Rameau plus accessible, se réalise en 1752 dans les Éléments de musique théorique et pratique suivant les principes de M. Rameau. Il est possible que Diderot y ait pris part. Mais cette tentative avorte, en partie par la faute de Rousseau. Celui-ci, je l'ai dit, ne manque que peu d'occasions de critiquer Rameau dans les articles qu'il écrit pour l'Encyclopédie, qui commencent à paraître avec le premier volume de 1751. Les choses ne s'arrangent pas en 1752, lorsque les troupes italiennes des Bouffes font salle pleine non dans les théâtres de la foire, comme à leur première visite, mais dans le haut lieu de la musique française, l'Opéra, sur la scène même des triomphes de Rameau. Les auditeurs se divisent alors en «coin de la reine», supporters de la musique italienne, et «coin du roi », défenseurs de la musique française. Peu après, Rousseau récidive, en publiant en 1753 sa Lettre sur la musique française, critiquée assez vertement. Rameau passe alors à l'attaque et publie ses Erreurs sur la musique dans l'"Encyclopédie" en 1755.

On pourrait émettre l'hypothèse selon laquelle la "perception des rapports» est une thèse associée à l'Encyclopédie, mais à ses débuts seulement. Elle semble en effet faire partie d'une tentative, de la part des encyclopédistes, d'élaborer, à partir des œuvres de Rameau, une esthétique qui leur est propre et qui correspond à leurs choix intellectuels. Cette esthétique pourrait opérer une synthèse entre l'intellect et les sensations, entre des "rapports» abstraits et la «perception ${ }^{16}$. La notion de la "perception des rapports» marque peut-être une différence qui se creuse entre Diderot et Rousseau. On en trouve un indice dans un des articles de Rousseau pour l'Encyclopédie, où il loue Diderot d'avoir abandonné les explications basées sur les formules mathématiques pour les cordes vibrantes:

Un écrivain judicieux, qui nous a donné nouvellement des principes d'Acoustique, laissant à part tous ces concours de vibrations, a rendu raison du plaisir que les

15. Voir le beau livre de D. Charlton, Opera in the Age of Rousseau: Music, Confrontation, Realism, Cambridge, Cambridge University Press, 2013.

16. Briseux distingue, dans ce contexte, perception et sensation. 
consonances font à l'oreille par la simplicité des rapports entre les sons qui les forment. Selon lui, le plaisir diminue à mesure que les rapports deviennent plus composés; et quand l'esprit ne les saisit plus, ce sont de véritables dissonances ${ }^{17}$.

Mais Rousseau, on le voit tout de suite, ne recule que pour mieux sauter: «il se trouve que [cette doctrine n'est] pas en tout d'accord avec l'expérience ${ }^{18}$. Il reconnaît néanmoins que la doctrine s'accorde bel et bien avec les divisions harmoniques simples; et qu'elle comporte le grand avantage de s'étendre «facilement à d'autres phénomènes qui se remarquent dans les beaux-arts ». Cette dernière phrase nous offre un indice important: Diderot tenterait d'élaborer avec «la perception des rapports» une esthétique qui, sous le régime de la raison et de la simplicité, s'appliquerait à tous les arts. Rousseau objecte que tout cela n'est pas démontré.

Lorsque Rousseau reprend son article «Consonance» dans le Dictionnaire de musique, il change son allusion à Diderot: celui-ci, d' "écrivain judicieux", devient «Le Philosophe», et se retrouve tributaire de Descartes pour sa pensée sur les rapports ${ }^{19}$. Un des ajouts que fait Rousseau nous livre une formule qui résume son objection à la «perception des rapports» comme explication de notre relation à la musique: "C'est une opération de l'esprit qu'ils prennent pour le principe du sentiment de l'harmonie $»^{20}$. L'harmonie pour Rousseau est donc un sentiment et non pas un calcul de l'intellect.

Nous avons vu que Rousseau attaque Rameau - et au même moment Diderot - pour la conséquence qui peut être tirée de leurs théories: pour Rousseau, l'harmonie ne représente pas la structure musicale - parfois inaudible - de ce qu'on entend; elle fait partie de la musique. La musique est par nécessité ce qui est audible, non pas ce qui est proportionnel à ce qui est audible. Ce n'est pas une "opération de l'esprit», une espèce de calcul inconscient.

Dans l'article «Tempérament» de son Dictionnaire de musique, Rousseau met le doigt, me semble-t-il, sur le problème. Rameau, dans certains de ses écrits, reconnaîtrait l'extrême labilité des sons, leur extrême dépendance au contexte sonore:

17. J.-J. Rousseau, art. «Consonance», in Encyclopédie ou Dictionnaire raisonné des sciences, des arts et des métiers, D. Diderot et J. Le Rond d'Alembert (éd.) ; édition en ligne: R. Morrissey (éd.), University of Chicago, ARTFL Encyclopédie Project, 2013, disponible à cette adresse: http://encyclopedie.uchicago.edu/. Les «Principes généraux d'acoustique» constituent le premier chapitre des Mémoires sur différents sujets de mathématiques de Diderot.

18. Ibid.

19. A. Charrak, «Rousseau et la musique: passivité et activité dans l'agrément», Archives de philosophie, t. 64, $\mathrm{n}^{\mathrm{0}} 2$ 2, 2001, p. 325-342.

20. J.-J. Rousseau, art. "Consonance», in Dictionnaire de musique, in OC V, p. 729. 
Il est bon d'observer, dit M. Rameau, que nous recevons des impressions différentes des Intervalles à proportion de leurs différentes altérations. Par exemple, la Tierce majeure, qui nous excite naturellement à la joie, nous imprime jusqu'à des idées de fureur quand elle est trop forte ${ }^{21}$.

Mais ailleurs dans ses écrits, Rameau tiendrait, selon Rousseau, « un tout autre langage», cherchant à fixer la relation entre les tons. Dans son Dictionnaire de musique, Rousseau nie catégoriquement, contre Rameau, qu'on puisse postuler «quelque identité entre les propriétés de la quantité abstraite et les sensations de l'ouïe ${ }^{22}$. Et d'une manière cinglante, Rousseau conclut à propos de Rameau: «la proportion lui sert pour introduire la Dissonance, et le défaut de proportion pour la faire sentir ${ }^{23}$. Tout cela constitue un ajout à l'article du quatrième volume de l'Encyclopédie de 1754, qui est beaucoup plus court.

Diderot est quant à lui plus hésitant, semble-t-il. La digression que je viens de faire du côté de Rousseau nous aidera à comprendre les deux Lettres de 1749 et 1751 . Très peu de temps après son «Mémoire» sur l'acoustique, il rédige la Lettre sur les aveugles. Dans les dernières pages, il expose ce qu'il est convenu d'appeler le "problème de Molyneux", qui porte sur la relation entre le toucher et la vision. Un homme aveugle de naissance, à qui la vision aurait été restaurée, saurait-il distinguer un globe d'un cube seulement en les regardant? Ou lui faudrait-il quand même les toucher pour savoir lequel des deux objets devant lui est la sphère et lequel le cube? Et Diderot de suggérer qu'il existe toute une part d'apprentissage dans la perception de l'espace. À l'encontre des philosophes anglais, Berkeley par exemple, Diderot insiste sur le côté social de cet apprentissage: il faut une certaine familiarité avec les objets se trouvant dans le champ visuel, il faut même comprendre leur fonction, pour pouvoir pleinement les reconnaître. La Lettre sur les aveugles ne s'en tient donc pas à une espèce de considération abstraite du toucher qui donnerait des informations sur la troisième dimension. Et dans la dernière section de cette lettre - qui à mon avis n'a jamais été vraiment éclairée -, Diderot envisagera même une espèce de déconstruction de la perception en imaginant un homme dont aucun des sens n'aurait de rapport avec les autres. Ce n'est là peut-être qu'un procédé d'hygiène mentale mais qui est tout de même troublant.

21. J.-J. Rousseau, art. «Tempérament», in Dictionnaire de musique, p. 1110.

22. "Cependant, dans un autre ouvrage, [Rameau] essaye d'en trouver le principe dans les rapports des nombres et les proportions harmonique et arithmétique, comme si il y avait quelque identité entre les propriétés de la quantité abstraite et les sensations de l'ouïe»; J.-J. Rousseau, art. «Dissonance», in Dictionnaire de musique, p. 766, remarque qui manque dans l'article publié dans le quatrième volume de l'Encyclopédie de 1754.

23. Ibid., p. 767. 
Car cette conjecture implique que Diderot envisagerait une perturbation de l'ordre expérientiel au niveau le plus profond, celui de la relation entre l'actif et le passif (et je viens d'apprendre que c'est une explication de la schizophrénie offerte de nos jours par certains psychiatres ${ }^{24}$ ). Diderot ne procède pas ici à une sorte de réduction à l'absurde, mais étend jusqu'aux confins du pensable les limites de la psychologie humaine. Ayant envisagé un désordre à l'échelle cosmique pour résoudre le problème de la régularité de notre perception, Diderot avance comme explication non une géométrie qui nous serait naturelle et inhérente, mais une explication sociale et anthropologique à la fois: notre instruction en géométrie.

À la fin de la Lettre sur les sourds et muets, Diderot place une série de paratextes - je ne commenterai ici ni le pourquoi ni le comment qui mériteraient pourtant une investigation plus serrée que celle reçue jusqu'ici. Parmi eux, la «Lettre à $\mathrm{M}^{\text {lle }}{ }^{* *}$ » décrit la "perception des rapports » comme un des "premiers pas de notre raison»- la raison qui perçoit des rapports simples et serait le principe fondamental du goût. On l'a vu, Rousseau a refusé cette simplicité. Quand on réfléchit aux exemples que donne Diderot dans cette lettre ${ }^{25}$, c'est-à-dire «les moulures, les filets, les galbes, les plinthes, les corniches, les panneaux, etc. ${ }^{26}$, ils semblent servir comme autant de raisons pour refuser le style rocaille. Ils ne sont, dit Diderot, que «des moyens suggérés par la nature pour s'écarter du rapport d'égalité et pour y revenir insensiblement $»^{27}$. Il fait de ces écarts / retours comme un mouvement, une promenade. Les rapports simples, dans les exemples qu'il donne ici, sont posés comme des effets de contraste, comme un point de retour stable après une digression et une divagation. Ici, ces rapports sont devenus les parties intégrantes d'une esthétique qui, moins fixée sur les proportions, est devenue plus ondoyante, plus accueillante aux effets du temps qui se déroule dans le mouvement.

Dans l'article «Beau», très proche en date de la «Lettre à $\mathrm{M}^{\text {lle } * * *}$ », le principe de la «perception des rapports» comme fondement de l'esthétique est longuement développé. Je n'en dirai ici que très peu de choses. Diderot nie, contre Batteux, mais également contre Rousseau, que le sentiment soit la base de notre plaisir dans la musique. Le «beau» prend son sens par rapport au contexte: Diderot nous offre toute une série de synonymes et

\footnotetext{
24. Je remercie Marc Jeanneret de m'en avoir fait la remarque.

25. M. Hobson et S. Harvey (éd.), «Introduction», in D. Diderot, Lettre sur les aveugles: à l'usage de ceux qui voient; Lettre sur les sourds et muets: à l'usage de ceux qui entendent et qui parlent, Paris, Flammarion, 200o, p. 144.

26. Ibid.

27. Ibid.
} 
d'antonymes pour nous le prouver; mais en même temps il affirme que la «perception des rapports» est la seule définition du beau qui ne soit pas sujette à la relativité dans l'espace ou dans le temps. Nous avons, dans cet article, d'une part l'indétermination de ces rapports:

C'est l'indétermination de ces rapports, la facilité de les saisir, et le plaisir qui accompagne leur perception, qui ont fait imaginer que le beau était plutôt une affaire de sentiment que de raison ${ }^{28}$.

D'autre part, nous avons leur rôle comme point d'ancrage de notre expérience du beau:

L'application de ce principe peut varier à l'infini, et ses modifications accidentelles occasionner des dissertations et des guerres littéraires: mais le principe n'en est pas moins constant. Il n'y a peut-être pas deux hommes sur la terre qui aperçoivent exactement les mêmes rapports dans un même objet, et qui le jugent beau au même degré; mais s'il y en avait un seul qui ne fût affecté des rapports dans aucun genre, ce serait un stupide parfait; et s'il y était insensible seulement dans quelques genres, ce phénomène décèlerait en lui un défaut d'économie animale; et nous serions toujours éloignés du scepticisme, par la condition générale du reste de l'espèce ${ }^{29}$.

Diderot insiste, contre Rousseau, sur le fait que c'est la raison, et non le sentiment, qui assure la régularité de notre expérience de ces rapports et de leur indétermination. L'économie animale et la condition générale de l'espèce imposent une régularité à nos réactions; nous avons donc là comme une esthétique structurée par la physiologie et la psychologie, une esthétique purement humaine, une esthétique en fait athée.

Rousseau, dans son Essai sur l'origine des langues, de date incertaine ${ }^{30}$, va plus loin que Diderot dans l'indétermination qu'il accorde aux rapports des sons dans la musique - indétermination qu'il refuse aux couleurs:

28. D. Diderot, Recherches philosophiques sur l'origine et la nature du beau, in LEW II, p. 494. Ce texte constitua d'abord l'article «Beau» de l'Encyclopédie, puis fut nommé "Traité du beau ", puis Recherches philosophiques sur l'origine et la nature du beau. Les variations dans le titre vaudraient sans doute une petite recherche, surtout parce qu'un paragraphe ressemble quelque peu à un paragraphe de l'article «Droit naturel» de Diderot, qui marque à la fois l'entrée sur la scène intellectuelle du terme volonté générale et le début de sa séparation d'avec Rousseau.

29. Ibid., p. 510.

30. Je tends à croire que cet Essai sur l'origine des langues diffère plus qu'on ne le dit du Discours sur les fondements et l'origine de l'inégalité de 1755, et que ses concepts mêmes se sont développés plus tard; voir M. Hobson, «Nexus Effectivus and Nexus Finalis: Causality in Rousseau's Discours sur l'inégalité and the Essai sur l'origine des langues" [1992], in Diderot and Rousseau: Networks of Enlightenment, K. E. Tunstall et C. Warman (éd. et trad. angl.), Oxford, Voltaire Foundation (SVEC), 2011, p. 225-250. 
Un son n'a par lui-même aucun caractère absolu qui le fasse reconnaître: il est grave ou aigu, fort ou doux, par rapport à un autre; en lui-même il n'est rien de tout cela. Dans le système harmonique, un son quelconque n'est rien non plus naturellement; il n'est ni tonique, ni dominant, ni harmonique, ni fondamental, parce que toutes ces propriétés ne sont que des rapports, et que le système entier pouvant varier du grave à l'aigu, chaque son change d'ordre et de place dans le système, selon que le système change de degré ${ }^{31}$.

Diderot me semble-t-il arrive à une opinion comparable dans sa pensée esthétique, mais à propos de la peinture et plus tard. Ici, j'esquisserai très brièvement ce qui, à mon avis, se passe. Dans le Salon de 1763, il parle d'un peintre, Deshays, qui va mourir assez jeune, et ne remplira donc pas tous les espoirs que Diderot, comme beaucoup d'autres, plaçait dans son talent:

Assemblez confusément des objets de toute espèce et de toutes couleurs, du linge, des fruits, des liqueurs, du papier, des livres, des étoffes et des animaux, et vous verrez que l'air et la lumière, ces deux harmoniques universels, les accorderont tous, je ne sais comment, par des reflets imperceptibles; tout se liera, les disparates s'affaibliront, et votre œil ne reprochera rien à l'ensemble. L'art du musicien qui, en touchant sur l'orgue l'accord parfait d'ut, porte à votre oreille les dissonants $u t, m i, s o l, u t, s o l, s i, r e ́, u t$, en est venu là ; celui du peintre n'y viendra jamais. C'est que le musicien vous envoie les sons mêmes, et que ce que le peintre broie sur sa palette, ce n'est pas de la chair, de la laine, du sang, la lumière du soleil, l'air de l'atmosphère, mais des terres, des sucs [...]. De là l'impossibilité de rendre les reflets imperceptibles des objets les uns sur les autres; il y a pour lui des couleurs ennemies qui ne se réconcilieront jamais. De là la palette particulière, un faire, un technique propre à chaque peintre. Qu'est-ce que ce technique? L'art de sauver un certain nombre de dissonances, d'esquiver les difficultés supérieures à l'art ${ }^{32}$.

Diderot, seul dans son cabinet, se laisse tenter par la divagation. Au lieu de Deshays, il parle comme s'il pensait à Chardin. Il montre comment, dans la réalité, la couleur des objets n'est pas fixe, mais varie selon les couleurs environnantes. Il continue en montrant comment les tons disparates s'affaiblissent et s'accordent sous l'effet des reflets et compare cet effet à celui des harmoniques des tons dans la musique. Mais la musique n'a pas d'analogue strict dans la peinture: elle utilise le son même, tandis que le peintre doit passer par les pigments, c'est-à-dire des substituts et non la chose même: il doit choisir. La «perception des rapports» comme description de notre réception de l'art est devenue une perception des «reflets imperceptibles»

31. J.-J. Rousseau, Essai sur l'origine des langues, in OC V, p. 420.

32. D. Diderot, Salon de 1763, in Salons, J. Seznec et J. Adhémar (éd.), Oxford, Clarendon Press, 1957, p. 217. 
que créent la lumière et l'air, «ces deux harmoniques universels». Le peintre n'en dispose pas directement, mais par médiation, en les recréant à travers les terres, les oxydes de métal, etc., qui se trouvent sur sa palette. Une peinture est, dit Diderot, un tissu de faussetés.

La "perception des rapports», qui a pris naissance dans l'acoustique et dans les relations géométriques des sons, ne joue ici, dirait-on, aucun rôle. Or, ce n'est pas le cas. Les rapports y sont encore, et la proportion également. Au lieu donc, de conclure à une esthétique de Diderot multiforme, on peut se demander si la "perception des rapports » ne fonctionne pas comme un fil rouge, mais souterrain.

De là la nécessité d'un certain choix [...]; encore après ce choix, [...] le meilleur tableau, le plus harmonieux, n'est-il qu'un tissu de faussetés qui se couvrent les unes les autres. Il y a des objets qui gagnent, d'autres qui perdent, et la grande magie consiste à approcher tout près de la nature et à faire que tout perde ou gagne proportionnellement; mais alors ce n'est plus la scène réelle et vraie qu'on voit, ce n'en est pour ainsi dire que la traduction ${ }^{33}$.

Les rapports sont devenus des reflets; externe dans le jeu de la lumière, interne dans notre vision d'un grand tableau. L'adverbe "proportionnellement" trahit, me semble-t-il, le fait que la théorie de la "perception des rapports" est toujours active.

Marian Hobson

Queen Mary University of London

33. Ibid. 